\title{
Remote-Queueing Multiple Access (RQMA): Providing Quality of Service for Wireless Communications
}

\author{
Norival R. Figueira* and Joseph Pasquale \\ \{norival, pasquale\}@cs.ucsd.edu \\ Computer Systems Laboratory \\ Center for Wireless Communications \\ Department of Computer Science and Engineering \\ University of California, San Diego \\ La Jolla, CA 92093-0114
}

\begin{abstract}
Providing quality-of-service (QoS) guarantees for wireless communications requires special consideration for the relatively large and time-varying bit error rates of wireless links, due to impairments that are difficult to predict. We describe a new wireless link access scheme called Remote-Queueing Multiple Access (RQMA), which allows seamless integration of scheduling disciplines proposed for wired networks that achieve end-to-end QoS guarantees. RQMA supports the flexible and efficient allocation of bandwidth for real-time sessions. Mobile stations send information about their backlogged packets to a base station which executes a scheduling discipline that provides delay bounds. Some minimum portion of the link bandwidth is reserved for the retransmission of packets that are not correctly received but whose deadlines are still not violated. For a fast fading channel with an average error rate of $10^{-2}$, RQMA is capable of delivering real-time traffic with a small average packet loss rate on the order of $10^{-5}$. Similar performance can be achieved for a slow fading channel if the average error rate is no more than $10^{-3}$.
\end{abstract}

\section{Introduction}

Packet-switching networks are increasingly being required to provide support for traffic types such as voice, video, and other kinds of interactive data. These traffic types, generally called real-time, require performance guarantees in terms of throughput, end-to-end delay, and packet loss rate. Most of the research on quality of service (QoS) in wired packet-switching networks has focused on resource reservation and scheduling within switches, without much concern for the bit error rate of the links since it is generally very small and predictable. However, wireless links have a relatively large and time-varying bit error rate due to impairments that are difficult to predict. How to properly integrate wireless links within a wired network that supports QoS guarantees is an important question.

* Norival R. Figueira is now with the Bay Architecture Lab, Bay Networks Inc., 4401 Great America Parkway, P.O. Box 58185, MS SC1-04, Santa Clara CA 95052-8185. His email address is norival @ baynetworks.com.

This work was supported by the UCSD Center for Wireless Communications.
We describe a new link-access scheme, Remote-Queueing Multiple Access (RQMA), that supports packet transmission over wireless links, allowing the use of scheduling disciplines that have been proposed for wired networks. Importantly, RQMA can be used with deadline-ordered [5] scheduling disciplines such as: Delay Earliest-Due-Date (Delay-EDD) [2], Jitter Earliest-Due-Date (Jitter-EDD) [15], VirtualClock [17], Leave-in-Time [4], and Rate-Function Scheduling (RFS) [6]. This allows a wireless link to be seamlessly integrated with a wired network that supports QoS guarantees.

RQMA assumes a microcell with one base station, called the base, and a number of mobile stations, called the mobiles. All communications are between a mobile and the base (mobiles do not communicate directly with other mobiles). Mobiles send information to the base about real-time packets that have recently arrived. Enough information is sent, in an efficient and reliable way, so that a scheduling discipline executed by the base can establish a transmission schedule in accordance with some QoS provision (such as delay bounds). The base then sends transmission permits to mobiles according to this schedule. Packets that are destined for mobiles within its cell are simply broadcast by the base.

To counteract the wireless link's channel errors, RQMA allows some portion of the link bandwidth to be reserved for the retransmission of real-time packets that are not correctly transmitted. Real-time packets are retransmitted until: (1) they are correctly received, or (2) their deadlines are violated, or (3) there is not enough link capacity to keep trying more retransmissions. This error control is in addition to the use of forward error correction (FEC), which by itself (i.e., by using even more FEC bits) is significantly less effective than their combination.

The remainder of this paper is organized as follows. Section 2 describes the basic algorithms of RQMA. In Section 3, the performance of RQMA is demonstrated through simulation experiments. Section 4 presents some possible extensions of RQMA. In Section 5, we discuss related work, and in Section 6 we present conclusions.

\section{Remote-Queneing Multiple Access}

Section 2.1 describes the basic algorithm of RQMA. Section 2.2 describes how RQMA deals with transmission errors. 


\subsection{RQMA Overview}

We define a session simply as a sequence of packets. A real-time session is one for which the packets have (transmission) deadlines. A continuous bit rate (CBR) session is one whose packets are generated at a constant rate, and whose deadlines are known a priori to be spaced at equal intervals. While CBR is actually a special case of real-time, we consider them separately, i.e., a session whose packets have deadlines is either CBR or real-time (anything other than CBR). Finally, a best-effort session is one for which packets do not have deadlines.

The time axis is divided into frames of length $T$ seconds. Each frame is subdivided into three fields: request, transmit, and backlog (Figure 1). The request field is subdivided into $r$ request slots and an acknowledgment (ack) subfield. The transmit field is subdivided into $t$ transmit slots, each composed of assign, data, and ack subfields. Finally, the backlog field is subdivided into $b$ backlog slots.

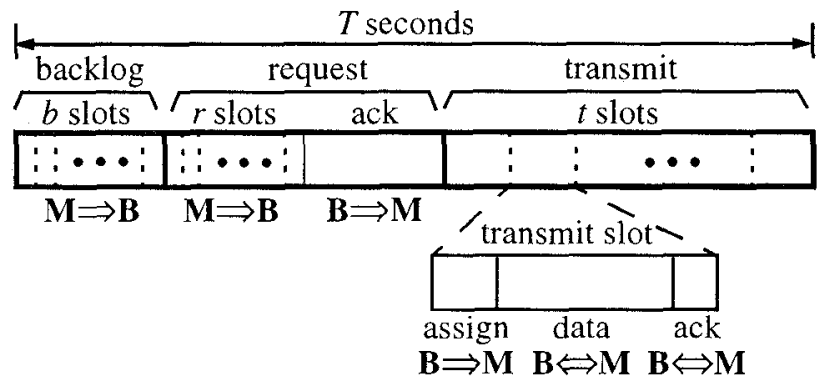

Figure 1: The structure of a frame. $M \Rightarrow B$ means transmission from mobile (M) to base station (B), and vice-versa for $B \Rightarrow M$. The result of the transmit field may depend on the backlog field of the same frame; hence, the backlog field comes at the beginning to give the base more time to process its contents before the first transmit slot assignment.

Mobiles send requests to the base to either establish real-time or CBR sessions, or to send one or more best-effort packets. There are four types of requests (described further in Section 2.1.1): Alloc_real-time, Alloc_CBR, Alloc_best-effort, and Cancel (applies to both real-time and CBR). Requests are sent in request slots using a random access protocol such as slotted Aloha. When the base successfully receives a request, it sends an acknowledgment in the request field's ack subfield.

Once a session has been established, the base decides when a mobile can send or receive the actual data packets of a session. Using a transmit slot's assign subfield, the base assigns the data subfield to a particular session, indicating which mobile is to make use of it. A mobile may only transmit a packet in the data subfield if such permission was given. The transmit slot's ack subfield is used to acknowledge successfully received ${ }^{1}$ data packets by a mobile or the base, depending on the direction of the transmission indicated in the transmit slot's assign subfield.

1. By "successfully received," we mean that correct data was recovered after FEC is processed at the receiver. In this paper, we assume that FEC processing occurs in parallel with the reception of a packet.
A backlog slot is associated with each established real-time session of a mobile. Backlog slots are used to inform the base about newly arrived packets of real-time sessions at a mobile. This information is used by the base's packet scheduling algorithm to determine when the mobile should transmit these packets.

Table 1 summarizes the contents of the various RQMA fields.

Table 1: Contents of RQMA Fields.

\begin{tabular}{|l|l|}
\hline \multicolumn{1}{|c|}{ Fields } & \multicolumn{1}{|c|}{ Contents } \\
\hline \hline Backlog slot & $\begin{array}{l}\text { sequence number of first, } \\
\text { deadline of last, number of } \\
\text { packets, new_info flag }\end{array}$ \\
\hline Request slot & $\begin{array}{l}\text { mobile identifier, type, } \\
\text { sequence last I rate l session } \\
\text { identifier ("l" means "or") }\end{array}$ \\
\hline Request: Ack subfield & r mobile identifiers \\
\hline Transmit: Assign subfield & $\begin{array}{l}\text { session identifier, sequence } \\
\text { number, direction, } \\
\text { first_of_frame }\end{array}$ \\
\hline Transmit: Data subfield & $\begin{array}{l}\text { data bits, deadline for } \\
\text { real-time/CBR I } \\
\text { piggy-back for best-effort }\end{array}$ \\
\hline Transmit: Ack subfield & \begin{tabular}{l} 
acknowledgment \\
\hline
\end{tabular} \\
\hline
\end{tabular}

Parameters $r, t$, and $b$ are separately configurable for different implementations of RQMA, and have the following implications. The number of backlog slots $b$ defines the maximum number of real-time sessions that can be established. The number of request slots $r$ is selected taking into consideration the maximum number of mobiles (usually, increasing $r$ decreases the average number of collisions of requests). The number of transmit slots $t$ is the prime contributor to the frame length and the overhead imposed by RQMA. A smaller value of $t$ implies a smaller frame length but a larger RQMA overhead. Larger values of $r$ and $b$ also imply a larger RQMA overhead. Section 3 provides a sample configuration for these parameters.

\subsubsection{Requests}

All requests carry a mobile identifier and a request type. Besides these parameters, the best-effort request also carries either the last sequence number of best-effort packets, the desired rate for a CBR session, or a session identifier for a real-time session.

Real-time allocation: An Alloc_real-time request is used by a mobile to establish a real-time session. After receiving (and acknowledging) an Alloc_real-time request, the base executes an admission control procedure required by its scheduling algorithm. The admission control procedure verifies that there are enough resources (e.g., link capacity) to accept this new real-time session without violating the QoS guarantees given to all other established real-time and CBR sessions. Once established, the base allocates a backlog slot number to the real-time session. Information exchange during the admission control 
procedure as well as informing a mobile about its backlog slot number allocation is carried out using a higher layer protocol (further discussion of such protocols is beyond the scope of this paper).

CBR allocation: An Alloc_CBR request is used when a mobile wants to establish a CBR session. Alloc_CBR requests are processed in the same way as Alloc_real-time requests. After accepting the session, the base regularly grants some transmit slots for the mobile to transmit CBR packets. Since the base knows the rate of packet transmission, CBR sessions do not use backlog slots.

Best-effort allocation: An Alloc_best-effort request is used by a mobile to transmit best-effort packets. After receiving (and acknowledging) an Alloc_best-effort request (which may request the transmission of multiple packets), the base station eventually allocates transmit slots for the transmission of these packets.

In this paper, we assume that a mobile station has only one uplink best-effort session, which is automatically established, and which is composed of all the best-effort traffic generated at the mobile. Thus, a best-effort request implicitly identifies this "session". This assumption is made to simplify the description and operation of RQMA; however, RQMA can be easily extended to deal with the general case of more than one best-effort session per mobile.

Cancel request: A mobile cancels a real-time or a CBR session by sending a Cancel request.

\subsubsection{Base Station Operation}

The base maintains three queues: a real-time/CBR queue, a best-effort queue, and an unreserved queue (Figure 2a). The real-time/CBR queue keeps track of real-time and CBR packets, the best-effort queue keeps track of best-effort packets, and the unreserved queue is used to deal with transmission errors (explained in Section 2.2.1). These queues keep track of local packets, which are stored at the base waiting to be transmitted to mobiles, and remote packets, which are stored at mobiles waiting to be transmitted to the base. Consequently, the contents of remote packets are not actually stored in these queues, but references to them are.

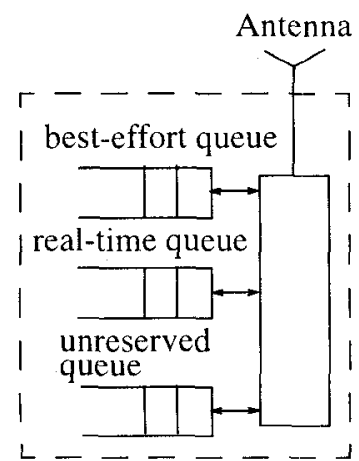

(a) Base

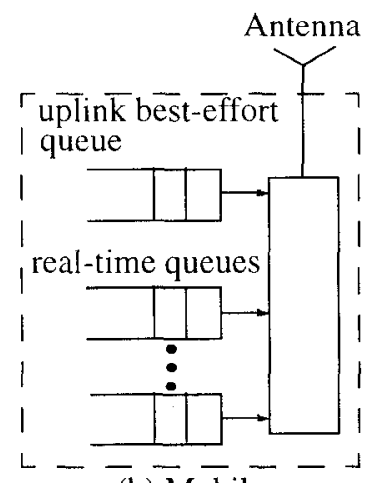

(b) Mobile
Figure 2: Queues at a (a) base station and (b) mobile station. The mobile's queues are used for uplink transmissions, whereas the base's queues are for both uplink and downlink.
The real-time/CBR queue is sorted according to packet deadlines. The deadlines of real-time packets are calculated by the base if the direction of the real-time session is from base to mobile; otherwise, they are calculated by the mobile and sent to the base using backlog slots. Deadlines of real-time packets are calculated based on some real-time scheduling discipline. The base also assigns deadlines to CBR packets, calculated based on the (fixed) rate of the session using the formula: deadline_of_next_packet $=$ deadline_of_previous_packet + packet_length / CBR_session_rate.

Local packets are queued for downlink transmission as soon as they arrive at the base. Remote real-time packets are queued for uplink transmission after the base receives arrival information in backlog slots. For remote $\mathrm{CBR}$ packets, queueing for uplink transmission is done periodically and automatically based on the known rates of the established CBR sessions. Finally, remote best-effort packets are queued for uplink transmission after the base receives alloc_best-effort requests.

The real-time/CBR queue is served using the earliest deadline first policy: transmit packets in increasing order of deadline, where ties are ordered arbitrarily. The base only serves the best-effort queue when the real-time/CBR queue is empty. ${ }^{2}$ RQMA does not define how the best-effort queue is scheduled. For example, one can use first-come first-served or round robin (for the latter, the base has a queue for each best-effort session).

To "serve" a packet, the base uses a transmit slot's assign subfield to indicate which mobile should make use of the corresponding data subfield, and the direction of transmission (mobile to base, or vice-versa). A mobile can transmit a packet in a data subfield only if permission was given by the base in the corresponding assign subfield. A packet is considered successfully transmitted only if the receiver sends an ack in the corresponding ack subfield.

\subsubsection{Mobile Station Operation}

A mobile maintains a queue for each of its uplink real-time sessions (a mobile can have more than one real-time session) and one queue for its uplink best-effort session (Figure $2 b$ ). The mobile listens to and inspects all the transmit slots' assign subfields to determine whether it should use the corresponding data subfield to transmit (or receive) a packet of data. The assign subfield is composed of a session identifier, the sequence number of the packet, direction of the transmission, and a binary flag called first-of-frame (used to compensate for transmission errors, and discussed in Section 2.2.1). The session identifier is unique among all mobiles; thus, there is no need to explicitly identify the mobile.

For each established real-time session, the mobile transmits (in every frame) the following information in the corresponding backlog slot (recall that a real-time session is allocated a backlog slot when the session is established): the sequence number of the first packet of that session that arrived during the previous frame, the number of packets of that ses-

\footnotetext{
2. In this case, real-time sessions must be rate controlled to avoid indefinite starvation of best-effort sessions. Alternatively, one could allocate some small part of the (wireless) link capacity to best-effort sessions and treat them as real-time to guarantee that they will not suffer from indefinite starvation.
} 
sion that arrived during the previous frame, the deadline of the last packet of that session that arrived during the previous frame, and the new info bit.

The binary flag new info is used to indicate if a backlog slot contains new information. New_info is set to 0 if there were no new packet arrivals during the previous frame. In this case, the other fields of the backlog slot contain the same information of the previously transmitted backlog slot. Otherwise, new_info is set to 1 indicating there were one or more new packet arrivals during the previous frame, and consequently, the rest of the backlog slot contains new information. The new_info flag is useful when backlog slots are lost due to transmission errors (see Section 2.2.2).

Every time the base receives a backlog slot with new_info equal to 1 , it includes references for all the (remote) packets that arrived during the previous frame in the real-time queue. The deadlines of all of these packets are set to the deadline of the one that last arrived (as indicated in the backlog slot). Since these packets are only available for transmission after the back$\log$ slot is received by the base, a mobile needs to add one frame length $T$ in the deadline calculations of all real-time packets (since the base will not know for at least one frame length what the new arrivals are). Otherwise, the scheduling algorithm executed at the base may not be able to guarantee that all real-time packets will be transmitted before their deadlines (assuming no transmission errors). Because of this, the delay bounds of all uplink real-time sessions are increased by one frame length. Note that the deadlines of downlink real-time packets do not need to be increased by one frame length since the base already knows about all the newly arrived (downlink) packets.

For uplink transmission of a best-effort packet, the mobile must ask the base for a transmit slot by sending an Alloc_best-effort request either in a request slot, or by piggy-backing the request in the uplink transmission of one of its best-effort packets before the next selected request slot arrives. A piggy-back request is considered successful if the data packet carrying the piggy-back request is acknowledged by the base. When the base receives a best-effort request, it assigns sequence numbers to all the new (remote) packets since the last best-effort request, and includes these packets in its best-effort queue.

\subsection{Transmission Errors}

RQMA assumes that some form of FEC is used to reduce the average packet error rate (see Section 3 for an example). However, FEC cannot be used exclusively without incurring excessive overhead. This section describes how RQMA deals with unrecovered transmission errors.

\subsubsection{Errors in Transmit Slots}

RQMA uses sequence numbers to deal with data transmission errors. Each session is assigned a sequence number integer variable that increments by one unit for every transmitted packet of that session.

A real-time or CBR session can be considered a numbered sequence of packets. The (transmission) sequence number of a real-time or CBR packet is equal to the number of the packet within the session. Real-time and CBR packets may be dropped either due to buffer overflows (although this should be avoided by the proper reservation of resources) or due to deadline violations. Thus, sequence numbers of dropped packets will be skipped. ARQ (automatic repeat request) protocols are not used for dropped real-time or CBR packets.

All real-time packets also carry their deadlines (deadlines of CBR packets are implicit). The deadline of a successfully received packet is used by the base or mobile to determine when previously missed packets are lost. When the deadline of a successfully received packet is about to expire, all packets with smaller sequence numbers are considered lost.

Real-time scheduling disciplines proposed for wired networks typically assume that all transmissions are successful: after the transmission of a packet, no retransmissions will occur. If retransmissions were possible, the scheduling discipline might not be able to insure QoS guarantees. To support retransmissions, while still maintaining our goal of using existing real-time scheduling disciplines, a separate session is established called the (real-time) retransmission session. This session reserves some part of the available bandwidth of the link to support the retransmission of real-time and CBR packets. ${ }^{3}$ Thus, if the transmission of a real-time or CBR packet is not successful, the packet is assumed to have just arrived as part of the retransmission session (i.e., assuming the deadline of the packet is not yet violated). A new deadline for this packet is calculated. If the new deadline is later than the original deadline, the packet is dropped. Otherwise, the packet is kept in the real-time/CBR queue with its original deadline, and can be retransmitted without violating the QoS guarantee of the original session. This works because loosening up the deadlines of the packets of a session (in this case, the retransmission session) does not affect the schedulability of a service discipline [5].

Besides the above retransmission scheme for real-time packets, a backup scheme can be used for real-time or CBR packets whose deadlines are not yet violated but cannot be considered for the retransmission session (i.e., because there is not enough reserved retransmission bandwidth). In this scheme, the base includes those packets in the unreserved queue (recall that this is one of the three queues maintained by the base). This queue is sorted by deadlines and works just like the real-time/ CBR queue. However, the unreserved queue is served as if it were a best-effort queue, i.e., it is served after the real-time/ $\mathrm{CBR}$ queue is emptied and in round-robin fashion among the base's other best-effort queues if any.

If a mobile does not receive an ack after transmitting a real-time or CBR packet, the mobile will not know if the base successfully received it. In this case, the mobile will have to wait until the deadline of the packet expires before it can delete the packet from its queue.

For best-effort packets, if a mobile does not receive an ack after transmitting, the following algorithm is used to delete old (transmitted) packets from its best-effort queue. The first-of-frame binary flag in a transmit slot's assign subfield is

\footnotetext{
3. RMQA does not define whether the reserved amount is fixed or not.
} 
used to indicate whether the slot is the first being used for the corresponding session in the present frame. If this flag is turned on, all of the session's packets that were transmitted with sequence numbers smaller than that of this slot's packet were received by the base and can be deleted from the mobile's queue.

Unlike real-time or CBR sessions, a sequence number can be used repeatedly until some best-effort packet is successfully delivered using this sequence number. Thus, a mobile (or base) is allowed to drop best-effort packets (e.g., due to buffer overflow) without changing the sequence number for the session. RQMA does not define which, if any, ARQ protocol should be used for dropped best-effort packets.

Finally, if the assign subfield of a transmit slot is not correctly received, a mobile cannot make use of the corresponding data subfield. This kind of error will generate missed data packets, and is addressed by the schemes above.

\subsubsection{Errors in Backlog Slots}

In a frame where a mobile has nothing new to inform about a real-time session, the mobile repeats the same information from the previous backlog slot with new_info set to 0 . This allows the base to miss a backlog slot that has new_info set to 1 , and still be able to serve the packets. The mobile does not keep track of which backlog slots the base was able to receive. However, the base must make the necessary adjustments to the deadlines of packets in case it misses a backlog slot, as we explain next.

The base keeps track of the number of consecutive missed backlog slots of a session. Once the base successfully receives a backlog slot, it computes the number of packets it may have missed (using the received sequence number), and assigns as the deadline of all the missed packets the received deadline. If the received backlog slot has new_info set to 1 , the new packets informed in this backlog slot are processed as usual.

If only one backlog slot was missed and the last received backlog slot has new_info set to 0 , the deadline assigned to the missed packets is the correct one. Otherwise (if either the number of missed backlog slots is greater than one or the last received backlog slot has new_info set to 1 ), the assigned deadline may be larger than the original ones. Since this may increase the delay bound of these packets, the base assigns an expiration time to all of these packets after which they will be dropped. The expiration time guarantees that these packets will not be delivered with a delay larger than the delay bound of the session, and is calculated assuming that the first missed backlog slot had new_info set to 1 . In this case, if the last received back$\log$ slot has new_info set to 1 , the expiration time is equal to the assigned deadline plus the "latency" of the scheduling discipline (discussed below) minus one frame length for every missed backlog slot. If the last received backlog slot has new_info set to 0 , the expiration time is equal to the previous amount plus one frame length.

The latency of a scheduling discipline, which depends on the discipline, is the maximum difference between the delay guarantee given to a packet and the deadline of the packet. For example, in Delay-EDD this amount is equal to zero, and in
VirtualClock this amount is equal to one packet length divided by the capacity of the link.

We also need to guarantee schedulability [5]. Thus, (remote) packets originated from missed backlog slots are assumed to be from the retransmission session (as defined in Section 2.2.1). Thus, their original deadlines are compared to their deadlines in the retransmission session. If a packet's original deadline is before its deadline in the retransmission real-time session, the packet is dropped.

The base needs a mechanism to deallocate backlog slots. RQMA assumes that the base periodically broadcasts data packets containing backlog slot confirmations and deallocations. A mobile can only use a backlog slot for a predefined interval of time after the last confirmation. If a backlog slot was deallocated, the mobile sends an acknowledgment in the back$\log$ slot, and stops further use of it. This ack allows the base to reassign the backlog slot before the predefined timeout period. This algorithm guarantees that a mobile will eventually release a backlog slot even if it does not receive a deallocation from the base. Note that a CBR session can be deallocated by the base by simply denying the allocation of data slots.

\section{Simulation Experiments}

In this section, we evaluate the performance of RQMA through simulation experiments. Since only uplink real-time sessions require the use of the backlog field, we only simulate uplink sessions.

\subsection{Traffic Source Models}

We use two kinds of traffic sources: ON-OFF and Poisson. $\mathrm{ON}$-OFF sources have been used extensively in recent studies $[4,16]$ since they can be used to model standard voice sources. In our simulations, all real-time sessions are modeled as ON-OFF traffic sources and all best-effort sessions are modeled as Poisson sources.

ON-OFF traffic sources: An ON-OFF traffic source is modeled here as a two-state Markov modulated process. In the ON state, packets are generated at fixed intervals of time $\tau$. In the OFF state, no packets are generated. The duration of the ON and OFF states are exponentially distributed with mean $a_{O N}$ and $\mathrm{a}_{\mathrm{OFF}}$, respectively. The number of packets generated in the ON state is approximated by a geometric distribution with mean $\mathrm{a}_{\mathrm{ON}} / \tau$.

We simulated ON-OFF traffic sources with $\mathrm{a}_{\mathrm{ON}}=352 \mathrm{~ms}$ and $\mathrm{a}_{\mathrm{OFF}}=18.5 \mathrm{~ms}$. Although a more typical value for $\mathrm{a}_{\mathrm{OFF}}$ for a standard voice source is $650 \mathrm{~ms}$ [16], we use $\mathrm{a}_{\mathrm{OFF}}=18.5 \mathrm{~ms}$ (also one of the values used in [16]) to increase the link utilization and, consequently, to limit the amount of spare bandwidth available. In our simulations, we set $\tau=13.25 \mathrm{~ms}$, which implies that the generation rate is $32 \mathrm{kbits} / \mathrm{s}$ in the ON state, since we use ATM-like packets of 424 bits.

Poisson traffic sources: The interarrival time of packets for these traffic sources is exponentially distributed. We use a value of $100 \mathrm{~ms}$ for the mean interarrival time to avoid large backlogs of best-effort packets, since our simulations try to allocate most of the capacity of the link to real-time sessions. 


\subsection{Scheduling Discipline}

We use VirtualClock [17] as the packet scheduling discipline at the base because it is simple and easy to implement, while still providing end-to-end delay bounds to real-time sessions given the reservation of a lower bound of bandwidth [3]. All real-time sessions reserved a rate of $32 \mathrm{kbits} / \mathrm{s}$ in the wireless link.

\subsection{Transmission Errors}

We use the two-state Gilbert-Elliot Markov model [1,8], which is widely used to describe fading channels. This model describes a stochastic sequential machine (SSM) that generates a binary output sequence. In this sequence, a 1 (one) indicates that one bit was transmitted with error, while a 0 (zero) indicates the correct transmission of a bit.

This SSM has two states: a "good state" and a "bad state." In the good state, the probability that the SSM will generate a 1 is $p_{0}$, while in the bad state this probability is $p_{1}$. The SSM makes a transition from the good state to the bad state with probability $p_{b}$, and vice-versa with probability $p_{g}$. Define the average error rate $P_{a v}$ as the probability that the next output of the SSM is equal to 1 (see [10] for a derivation):

$$
P_{a v}=\left(\frac{p_{g}}{p_{b}+p_{g}}\right) p_{0}+\left(\frac{p_{b}}{p_{b}+p_{g}}\right) p_{1} .
$$

We simulated two kinds of channels: a slow fading channel and a fast fading channel. The fading parameters we use are similar to those used in [10]. For the slow fading channel: $p_{b}=10^{-6}$ and $p_{g}=3 \times 10^{-6}$. For the fast fading channel: $p_{b}=0.2$ and $p_{g}=0.6$. For both kinds of channels, $p_{0}=10^{-6}$ and $p_{1}=3.9997 \times 10^{-2}$. This results in a fairly high average error rate $P_{a v}$ of $10^{-2}$ for both channels.

\subsection{Forward Error Correction}

FEC is generally used to reduce errors in wireless communications; we use FEC in our simulations of RQMA. We use the Bose-Chaudhuri-Hocquenghem $(\mathrm{BCH})$ codes and the tables in [12] to calculate the number of FEC (overhead) bits. To simplify our simulations, we assumed that all errors are either corrected or detected.

We experimentally determined the point at which increasing the number of FEC bits, which reduces the usable bandwidth, produced diminishing returns in reducing the packet loss rate. Determining the number of FEC bits is complicated in that some frame bits are more important than others, and so one must also determine which frame bits they should correct. The complete study is beyond the scope of this paper; we simply present the final results. For more information, we refer the reader to [7], where we present an analysis on how the number of FEC bits for data packets was determined.

Table 2 shows the length of all the RQMA parameters and the number of FEC bits used in the simulations.
Table 2: Parameters Used in Simulation Experiments.

\begin{tabular}{|l|l|l|l|}
\hline \multicolumn{1}{|c|}{ Field } & Parameter Sizes (bits) & $\begin{array}{c}\text { FEC Bits } \\
\text { (Error- } \\
\text { corrected Bits) }\end{array}$ & $\begin{array}{l}\text { Total } \\
\text { Bits }\end{array}$ \\
\hline Backlog Slot & $\begin{array}{l}\text { sequence first (24), } \\
\text { number of packets (8), } \\
\text { deadline last (16), } \\
\text { new_info_slot (1) }\end{array}$ & $54(8)$ & 103 \\
\hline Request Slot & $\begin{array}{l}\text { mobile identifier (8), } \\
\text { type (2), } \\
\text { sequence last 1 rate (24) }\end{array}$ & $12(2)$ & 46 \\
\hline $\begin{array}{l}\text { Request: } \\
\text { Ack subfield }\end{array}$ & $\begin{array}{l}\text { mobile identifier (8) } * r \\
\text { (r=20) }\end{array}$ & $15(2)$ & 175 \\
\hline $\begin{array}{l}\text { Transmit: } \\
\text { Assign } \\
\text { subfield }\end{array}$ & $\begin{array}{l}\text { session identifier (9), } \\
\text { sequence number (24), } \\
\text { direction (1), } \\
\text { first_of_frame (1) }\end{array}$ & $45(7)$ & 80 \\
\hline $\begin{array}{l}\text { Transmit: } \\
\text { Data subfield }\end{array}$ & $\begin{array}{l}\text { data bits (424), } \\
\text { deadline 1 } \\
\text { piggy-back } \\
\text { best-effort (24) }\end{array}$ & $91(10)$ & 15 \\
\hline $\begin{array}{l}\text { Transmit: } \\
\text { Ack subfield }\end{array}$ & \begin{tabular}{l} 
ack (7) \\
\hline
\end{tabular}
\end{tabular}

\subsection{Frame and Link Parameters}

We assumed a microcell with a radius of $450 \mathrm{~m}$, and a link capacity of $2 \mathrm{Mbits} / \mathrm{s}$. We configured our frames to contain 40 backlog slots, 20 request slots, and 100 transmit slots. To prevent uplink signals from different mobiles from overlapping, a guard time must be provided for each separate uplink communication. The guard time must be at least equal to the maximum difference in the round-trip radio propagation delay between any two mobiles in the same cell, which is approximately $3 \mu \mathrm{s}$ given our microcell size. Finally, we use 10 bits for synchronization of packets. Given these parameters, the frame time is approximately $37 \mathrm{~ms}$.

\subsection{Simulation Results}

We present the results of an experiment that uses 33 mobiles, 33 best-effort sessions, and 25 real-time sessions. Figure 3 shows the percentage of real-time packets that are lost, i.e., that are not successfully transmitted before their deadlines, versus the amount of bandwidth that is reserved for the retransmission of real-time packets. Recall that the channel's average error rate is $10^{-2}$.

Figure 3 expresses the reserved retransmission rate in terms of the available capacity of the link, i.e., the amount of bandwidth that is left for the transmission of payload data after all the overhead is taken into account. The available capacity is about $1.15 \mathrm{Mbits} / \mathrm{s}$, of which about $30.4 \%$ (or $350 \mathrm{Kbits} / \mathrm{s}$ ) is available for retransmissions. The rest of the available bandwidth (about $800 \mathrm{Kbits} / \mathrm{s}$ ) is reserved for the 25 real-time sessions. To isolate the contribution of the bandwidth reservation for retransmission of real-time packets, the experiments shown in Figure 3 are for a base station that is not using the unreserved queue (as defined in Section 2.1.2). 
For the fast fading channel, bandwidth reservation for retransmission provides a good reduction in the packet loss rate of $10^{-5}$ (or $10^{-3} \%$ as shown in Figure $3 \mathrm{a}$ ). However, for the slow fading channel, bandwidth reservation for retransmission did not provide a reduction in the packet loss rate even at high levels of reservation. This is because retransmissions are ineffective when the channel fades for long periods of time, as does our simulated slow fading channel. In this case, real-time packets are dropped (because their deadlines expire) before they can be successfully transmitted. While RQMA cannot deal with this problem, we expect that any access protocol that is exposed to this kind of slow fading channel and high average channel error rate will experience a similar problem.

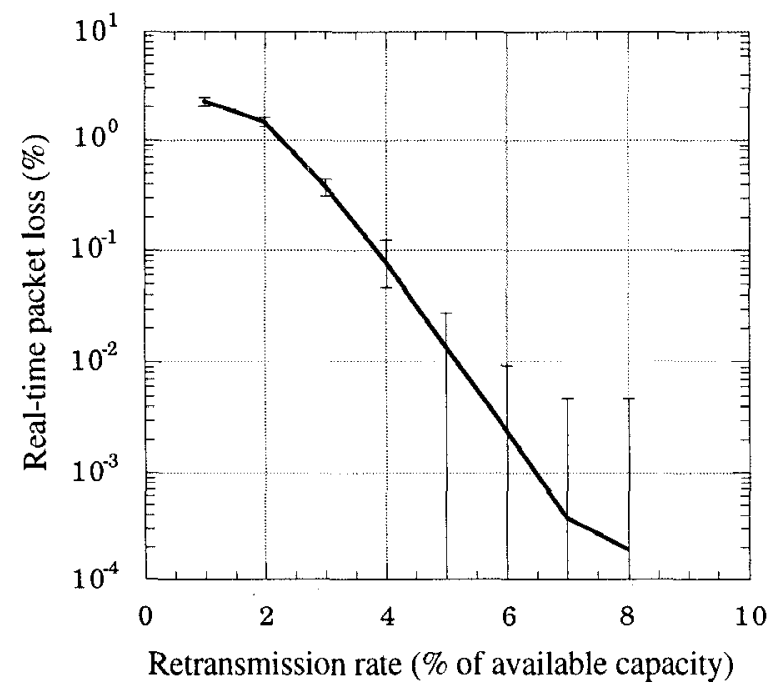

(a) Fast fading

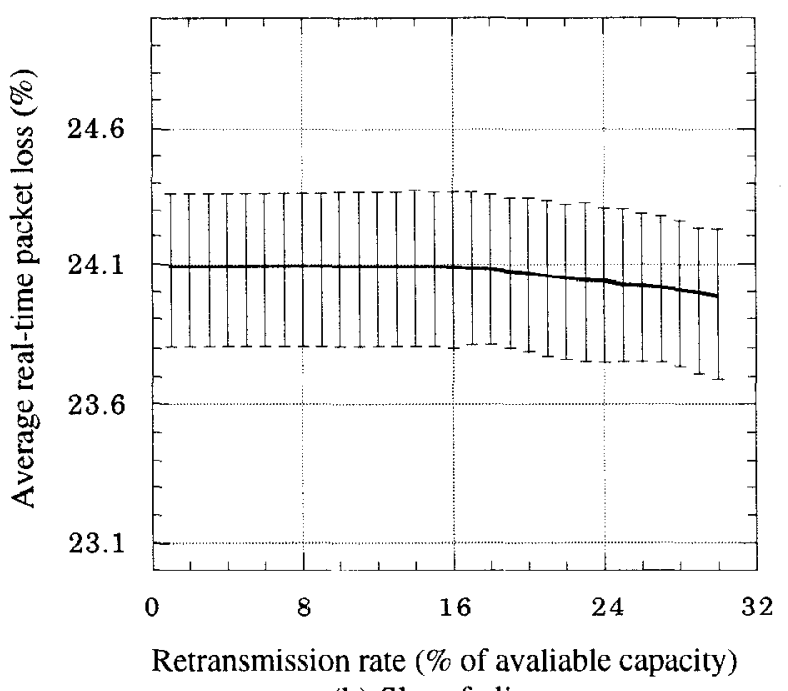

(b) Slow fading

Figure 3: Average real-time packet loss (as a percent of all packets) versus the reserved retransmission rate (as a percent of available capacity) for (a) a fast fading channel, and (b) a slow fading channel, with a channel average error rate of $10^{-2}$. The bars indicate the maximum and the minimum packet loss over all real-time sessions.
In [7], we carried out similar experiments for channels with lower average error rates, ranging from $10^{-3}$ to $10^{-5}$. In these cases, the results were as good as (and generally much better than) those in Figure 3a, for both fast and slow fading channels. Under these better conditions, RQMA is capable of delivering real-time traffic with a reasonably small average packet loss rate, on the order of $10^{-5}$.

\section{Extensions}

\subsection{Logical Frames}

Since the number of real-time backlog slots in a frame is finite, the maximum number of real-time sessions that can share a base is also finite. To allow the number of real-time sessions to exceed the number of backlog slots in a frame, one can associate real-time sessions with logical frames instead of physical frames. A logical frame is composed of an integral number of consecutive physical frames.

For example, if logical frames are composed of two physical frames, half of the logical frames beginning at odd physical frame boundaries and the other half beginning at even physical frame boundaries, we can have twice as many real-time sessions sharing the base. However, this scheme has the drawback that logical frames are longer than physical frames. This can be resolved by using a hierarchy of logical frames where the maximum number of logical frames that begins at any given physical frame is at most equal to the number of backlog slots of a physical frame. In this case, sessions can choose from a set of logical frames with various lengths. For example, a backlog field with two backlog slots can be used for three logical frames: one logical frame with one physical frame and two logical frames with two physical frames each (see Figure 4).

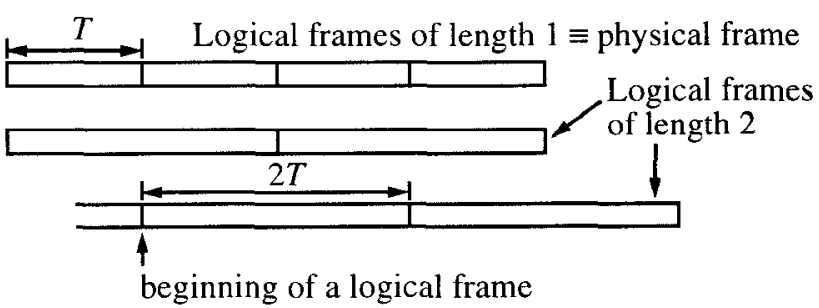

Figure 4: A hicrarchy of logical frames.

\subsection{Time Slots, Codes, and Frequency Slots}

This paper presents RQMA based on time slot allocation. However, nothing in RQMA dictates that these slots must represent time. Consequently, RQMA can easily be generalized to allocate frequency slots or codes (as in CDMA).

\section{Related Work}

RQMA builds on ideas found in Distributed-Queueing Request Update Multiple Access (DQRUMA) [11] and the work described in [14]. In DQRUMA, mobiles send transmission requests to the base, which broadcasts transmission permits. RQMA uses a similar request-permission scheme. 
However, RQMA differs by providing explicit support for real-time and CBR sessions; once these sessions are set up, packet delivery does not involve a random access protocol. Also, RQMA uses backlog slots for real-time sessions. A real-time session is allocated a backlog slot in every frame to allow it to send information about its newly arrived packets, which is used by the base's scheduling discipline to determine the transmission schedule of real-time packets and support performance guarantees.

Raychaudhuri and Wilson describe a "dynamic TDMA" scheme [14], where a TDMA frame is subdivided into request slots and message slots. Each message slot provides for the transmission of an ATM-like packet. Request slots are comparatively short and are used for initial access in slotted ALOHA contention mode. RQMA uses a similar framing structure, and assumes ATM-like packets. However, although RQMA defines a frame structure to allow for periodic request intervals, it is not based on TDMA since all data packet transmissions are explicitly controlled and assigned by the base in every message slot, leading to a more flexible bandwidth allocation that increases efficiency.

RQMA can also be compared to Packet Reservation Multiple Access (PRMA) [9] [13], which supports "periodic" (voice) traffic and "random" (data) traffic sources. Periodic sources contend for wireless channel time slots using random access, and then reserve future slots (similar to RQMA CBR sessions). However, unlike RQMA, PRMA does not support more general real-time traffic, where packets may have arbitrary deadlines and must be scheduled despite distributed information.

\section{Conclusions}

We presented a new scheme called Remote-Queueing Multiple Access (RQMA) that supports QoS guarantees over wireless links. The novel ideas in RQMA lie in how real-time (and CBR) sessions are supported, and how it deals with errors. For real-time sessions, RQMA uses a backlog field so that allocation of link bandwidth is flexible and efficient. Regarding errors, some minimum portion of the link bandwidth is reserved for the retransmission of packets that are not correctly received but whose deadlines are still not violated.

We presented the basic algorithms of RQMA and presented a performance evaluation of RQMA through simulation experiments. RQMA is capable of delivering real-time traffic with a small average packet loss rate (on the order of $10^{-5}$ ) for fast or slow fading channels with an average error rate of $10^{-3}$ or lower. Even with an average error rate of $10^{-2}$, the retransmission scheme of RQMA is still able to reduce the average packet loss rate to $10^{-5}$ for a fast fading channel. However, for a slow fading channel with an average error rate of $10^{-2}$, neither FEC in data packets nor retransmission of packets are able to effectively reduce the average packet loss rate. This is because the slow fading channel fades for long periods of time, and real-time packets are dropped (because their deadlines expire) before they can be successfully transmitted.

Based on these results, we believe that RQMA is a feasible solution for integrated wireless/wired networks that carry real-time traffic.

\section{References}

[1] E. O. Elliot, "Estimates of Error Rates for Codes on Burst-Noise Channels," Bell System Technical Journal, Vol. 42, pp. 1977-1997, September 1963.

[2] D. Ferrari and D. Verma, "A Scheme for Real-Time Channel Establishment in Wide-Area Networks," IEEE Journal on Selected Areas in Communications, Vol. 8, No. 4, pp. 368-379, April 1990.

[3] N. R. Figueira and J. Pasquale, "An Upper Bound on Delay for the VirtualClock service Discipline," IEEE/ACM Transactions on Networking, Vol. 3, No. 4, pp. 399-408, August 1995.

[4] N. R. Figueira and J. Pasquale, "Leave-in-Time: A New Service Discipline for Control of Real-Time Communications in a Packet-Switching Network," Proc. ACM SIGCOMM '95, pp. 207-218, August 1995.

[5] N. R. Figueira and J. Pasquale, "A Schedulability Condition for Deadline-Ordered Service Disciplines," IEEE/ACM Transactions on Networking, Vol. 5, No. 2, pp. 232-244, April 1997.

[6] N. R. Figueira and J. Pasquale, "Rate-Function Scheduling," Proceedings IEEE INFOCOM '97, pp. 1065-1074, April 1997.

[7] N. R. Figueira and J. Pasquale, "Remote-Queueing Multiple Access (RQMA): Providing Quality of Service for Wireless Communications," Center for Wireless Communications Technical Report, U. C. San Diego, January 1998

[8] E. N. Gilbert, "Capacity of a Burst Noise Channel," Bell System Technical Journal, Vol. 39, pp. 1253-1265, September 1960.

[9] D. J. Goodman, R. A. Valenzuela, K. T. Gayliard, and B. Bamamurthi, "Packet Reservation Multiple Access for Local Wireless Communications," IEEE Transactions on Communications, Vol. 37, pp. 885-890, August 1989.

[10] N. Guo and S. D. Morgera, "Frequency-Hopped ARQ for Wireless Network Data Services," IEEE Journal on Selected Areas in Communications, Vol. 12, No. 8, pp. 1324-1337, October 1994.

[11] M. J. Karol, Z. Liu, and K. Y. Eng, "Distributed-Queueing Request Update Multiple Access (DQRUMA) for Wireless Packet (ATM) Networks," Proc. International Conference on Communications, pp. 1224-1231, June 1995.

[12]S. Lin, "An Introduction to Error-Correcting Codes," Englewood Cliffs, Prentice-Hall, New Jersey, 1970.

[13] P. Narasimhan and R. D. Yates, "A New Protocol for the Integration of Voice and Data over PRMA," IEEE Journal on Selected Areas in Communications, Vol. 14, No. 4, pp. 623-631, May 1996.

[14]D. Raychaudhuri and N. D. Wilson, "ATM-Based Transport Architecture for Multiservices Wireless Personal Communication Networks," IEEE Journal on Selected Areas in Communications, Vol. 12, No. 8, pp. 1401-1414, October 1994

[15] D. Verma, H. Zhang, and D. Ferrari, "Delay Jitter Control for Real-Time Communication in a Packet Switching Network," Proc. IEEE TriCom '9l, pp. 35-43, April 1991.

[16] D. Yates, J. Kurose, D. Towsley, and M. G. Hluchyj, "On Per-session End-to-End Delay Distributions and the Call Admission Problem for Real-Time Applications with QoS Requirements," Proc. ACM SIGCOMM '93, pp. 2-12, September 1993.

[17] L. Zhang, "VirtualClock: A New Traffic Control Algorithm for Packet Switching Networks," ACM Transactions on Computer Systems, Vol. 9, No. 2, pp. 101-124, May 1991. 